\title{
The effect of the interaction between the minority phase droplets on the nucleation behavior during the liquid-liquid phase transformation
}

\author{
ZHAO JiuZhou', LI HaiLi \& HE Jie \\ Institute of Metal Research, Chinese Academy of Sciences, Shenyang 110016, China
}

The microstructure evolution during the liquid-liquid phase transformation of $\mathrm{Al}-\mathrm{Pb}$ alloy was calculated. The numerical results indicate that the interaction between the minority phase droplets has effect on the nucleation process of the droplets, and the effect increases with the cooling rate and the content of $\mathrm{Pb}$.

liquid-liquid phase transformation, nucleation, microstructure development

Liquid-liquid phase transformation occurs when a homogeneous, single phase liquid is cooled into the miscibility gap in the liquid state. Generally the liquid-liquid phase transformation begins with the nucleation of the minority phase droplets. These droplets grow then by the diffusion of solute in the matrix. The nucleation and diffusional growth processes dominate the formation of the solidification microstructure of immiscible alloys under the rapid solidification conditions. A fine dispersion of particles can be obtained by using the fast cooling technique ${ }^{[1-7]}$. Recently great attention has been paid to the study of the solidification of immiscible alloys ${ }^{\left[{ }^{-12]}\right.}$. But commonly the microstructure evolution during a cooling was investigated by neglecting the interaction between the minority phase droplets. The objective of this paper is to uncover the effect of the interaction between the minority phase droplets on the dispersoid formation in a rapidly solidified immiscible alloy, especially on the nucleation behavior during the liquid-liquid phase transformation.

\section{Model theory}

The radius distribution function of the minority phase droplets obeys ${ }^{[13]}$

$$
\frac{\partial f}{\partial t}+\frac{\partial}{\partial R}\left(\frac{\mathrm{d} R}{\mathrm{~d} t} f\right)=\left.\frac{\partial I}{\partial R}\right|_{R=R^{*}}
$$

Received February 11, 2007; accepted May 17, 2007

doi: $10.1007 / \mathrm{s} 11433-007-0037-7$

†Corresponding author (email: jzzhao@imr.ac.cn)

Supported by the National Natural Science Foundation of China (Grant Nos. 50395104, 50671111 and 50620130095) 


$$
I=N_{0} O \Gamma Z \exp \left[-\frac{\Delta G_{\mathrm{c}}}{k_{\mathrm{B}} T}\right],
$$

where $f(R, t)$ is the droplet radius distribution function. It is so defined that $f \cdot \mathrm{d} R$ gives the number of droplets per unit volume in a size class $R$ to $R+\Delta R$ at time $t$. $I$ is the nucleation rate of the minority phase droplets. $N_{0}=\left[X_{\mathrm{me}} \Omega_{\mathrm{Pb}}+\left(1-X_{\mathrm{me}}\right) \Omega_{\mathrm{Al}}\right]^{-1}, O=4 n_{\mathrm{c}}^{2 / 3}, \Gamma=6 D / \lambda, Z=\left(\frac{\Delta G_{\mathrm{c}}}{3 \pi k_{\mathrm{B}} T n_{\mathrm{c}}^{2}}\right)^{1 / 2}$, $\Delta G_{\mathrm{c}}=\frac{16}{3} \pi \frac{\sigma^{3}}{\Delta G_{\mathrm{V}}^{2}}$, where $\Omega_{\mathrm{Al}}$ and $\Omega_{\mathrm{Pb}}$ are the atomic volumes of component $\mathrm{Al}$ and $\mathrm{Pb}, X_{\mathrm{me}}$ is the mole fraction of the solute in the matrix liquid, $n_{\mathrm{c}}$ is the number of atoms in a MPD of the critical nucleus radius, $\lambda$ is the average jump distance of atoms due to diffusion, $k_{\mathrm{B}}$ is Boltzmann's constant, $\sigma$ is the interfacial energy between the two liquids, $\Delta G_{\mathrm{V}}$ is the gain in the free energy per unit volume on nucleation, $\frac{\mathrm{d} R}{\mathrm{~d} t}$ is the diffusional growth rate of the droplet.

The diffusional growth rate can be calculated by eq. (3) if the interaction between the concentration fields of droplets is negligible (i.e. for an isolated droplet):

$$
\frac{\mathrm{d} R}{\mathrm{~d} t}=D \frac{C_{\mathrm{m}}-C_{\mathrm{I}}}{C_{\beta}-C_{\mathrm{I}}} \frac{1}{R},
$$

where $C_{\mathrm{m}}$ is the mean field concentration in the matrix liquid, $C_{\beta}$ the concentration of the liquid within the droplet, and $C_{\mathrm{I}}$ the concentration of solute in the matrix at the inter-phase boundary.

Assuming that the interface of a growing droplet is always in local equilibrium, the interfacial composition depends on the particle radius according to the Gibbs-Thomson relation ${ }^{[14]}$.

$$
C_{\mathrm{I}}(R, t)=C_{\infty} \exp \left(\frac{\alpha_{\mathrm{s}}}{R}\right),
$$

where $C_{\infty}$ is the equilibrium composition at a flat interface boundary, $\alpha_{\mathrm{s}}=\left(2 \sigma \Omega_{\mathrm{d}} / k_{\mathrm{B}} T\right)$ is the capillary length, and $\Omega_{\mathrm{d}}$ is the atomic volume in the droplet.

In fact, the isolated droplet assumption is only applicable for a system with a very low volume fraction or number density of the minority phase droplets. Generally the interaction between the droplets plays an important role in a practical system. Marqusee ${ }^{[15]}$ derived the diffusional growth rate of a particle by taking into account the effect of the volume fraction of the particles in the system:

$$
\frac{\mathrm{d} R}{\mathrm{~d} t}=D \frac{C_{\mathrm{m}}-C_{\mathrm{I}}}{C_{\beta}-C_{\mathrm{I}}} \frac{1}{R}\left[1+R(4 \pi n\langle R\rangle)^{1 / 2}\right] .
$$

The concentration of the minority phase droplets changes with temperature during the liquidliquid phase transformation. Assuming that the composition of the minority phase droplets varies always according to the phase diagram, the droplet growth rate derived respectively from eqs. (3) and (5) are ${ }^{[8]}$

$$
\begin{gathered}
\frac{\mathrm{d} R}{\mathrm{~d} t}=D \frac{C_{\mathrm{m}}-C_{\mathrm{I}}}{C_{\beta}-C_{\mathrm{I}}} \frac{1}{R}+\frac{R}{3} \frac{\left(\mathrm{d} C_{\beta} / \mathrm{d} T\right) /(\mathrm{d} T / \mathrm{d} t)}{C_{\beta}-C_{\mathrm{I}}} \\
\frac{\mathrm{d} R}{\mathrm{~d} t}=D \frac{C_{\mathrm{m}}-C_{\mathrm{I}}}{C_{\beta}-C_{\mathrm{I}}} \frac{1}{R}\left[1+R(4 \pi n\langle R\rangle)^{1 / 2}\right]+\frac{R}{3} \frac{\left(\mathrm{d} C_{\beta} / \mathrm{d} T\right) /(\mathrm{d} T / \mathrm{d} t)}{C_{\beta}-C_{\mathrm{I}}}
\end{gathered}
$$




\section{Numerical results and discussion}

Calculations were carried out for $\mathrm{Al}-\mathrm{Pb}$ alloy. The main parameters used in the calculation are listed in Table 1. The numerical results show that the alloy becomes supersaturated when it is cooled into the miscibility gap (see Figure 1). The liquid-liquid phase transformation takes place when the supersaturation arrives at a critical value. During the period of the nucleation, the droplet number density increases rapidly (see Figure 2).
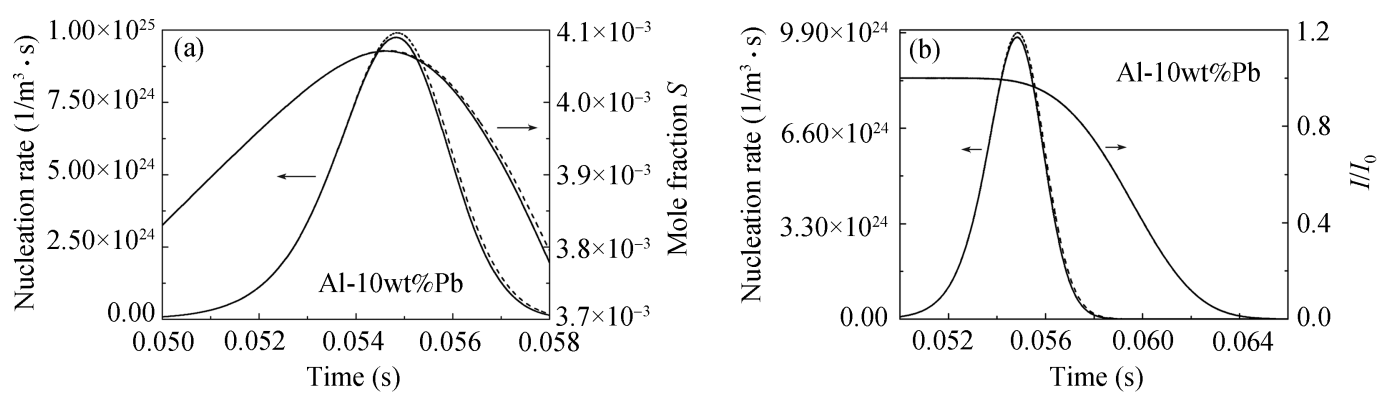

Figure 1 The supersaturation of the matrix liquid $\left(S=C_{\mathrm{m}}-C_{\infty}\right)$ and the nucleation rate $\left(I / I_{0}\right)$ of the minority phase droplets. The dashes and $I_{0}$ are the results calculated by neglecting the interaction between droplets.

Table 1 Main parameters used in the calculation

\begin{tabular}{lc}
\multicolumn{1}{c}{ Parameter } & Value \\
\hline$T_{\mathrm{m}}$ (Monotectic reaction temperature $)\left({ }^{\circ} \mathrm{C}\right)$ & 659.6 \\
$T_{\mathrm{c}}($ Critical temperature $)\left({ }^{\circ} \mathrm{C}\right)$ & 1244.4 \\
$D\left(\mathrm{~m}^{2} / \mathrm{s}\right)$ & $5 \times 10^{-9}(T+273)^{2} /\left(T_{\mathrm{m}}+273\right)^{2}$ \\
$\sigma\left(\mathrm{J} / \mathrm{m}^{2}\right)$ & $0.12 \times\left(1-T / T_{\mathrm{c}}\right)^{1.26}$ \\
$\Omega_{\mathrm{Al}}\left(\mathrm{m}^{3} /\right.$ atom $)$ & $166 \times 10^{-29}$ \\
$\Omega_{\mathrm{Pb}}\left(\mathrm{m}^{3} /\right.$ atom $)$ & $3.02 \times 10^{-29}$ \\
$\lambda(\mathrm{m})$ & $2.67 \times 10^{-10}$ \\
$k_{\mathrm{B}}(\mathrm{J} / \mathrm{K})$ & $1.3807 \times 10^{-23}$
\end{tabular}

The effect of the interaction between the droplets on the liquid-liquid phase transformation can be seen by comparing the nucleation rates calculated by using eq. (6a) or (6b) as the growth rate controlling equation. The interaction between droplets has a negligible effect on the nucleation behavior of droplets in the early period of nucleation because both the nucleation rate and the number density of the minority phase droplets are lower, and the interaction between droplets is weaker (see Figure 1). The effect of the interaction increases with the number density or the volume fraction of the

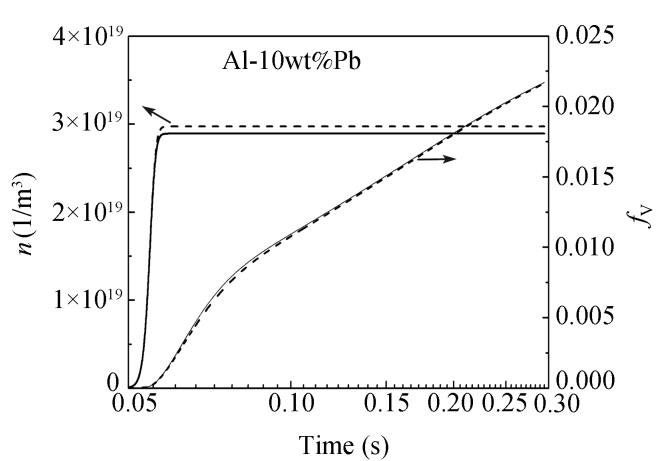

Figure 2 The number density $(n)$ and the volume fraction $\left(f_{\mathrm{V}}\right)$ of the minority phase droplets. The dashes are the result calculated by neglecting the interaction between droplets. 
droplets. A higher volume fraction of the minority phase droplets causes a larger growth rate of the droplets, a higher decreasing rate of the supersaturation and, therefore, a lower nucleation rate in the later stage of the nucleation (see Figure 1(b)) and a lower number density of the droplets (see Figure 2).

Figure 3 shows the variation of the number density and the volume fraction of the minority phase droplets with the cooling rate. For an alloy of given composition, the effect of the interaction between the droplets on the nucleation increases with the cooling rate, but shows an asymptotic tendency. This is because that the cooling rate has two opposite effects. A higher cooling rate leads to a higher nucleation rate and a higher growth rate of droplets, which favors the increase of the volume fraction of droplets during the period of nucleation. On the other hand, the nucleation lasts a shorter period of time under a higher cooling rate, which is unfavorable for the increase of the volume fraction of droplets.

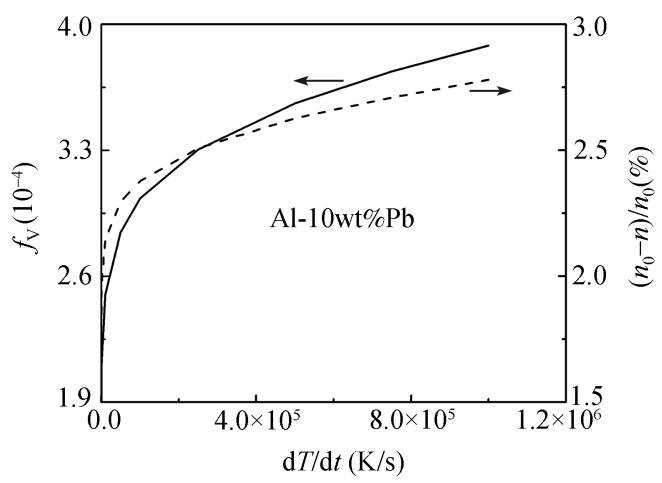

Figure 3 The volume fraction of the minority phase droplets as a function of the cooling rate when $n / n_{0}$ arrives at 0.9 . $n_{0}$ is the number density calculated by neglecting the interaction between the droplets.

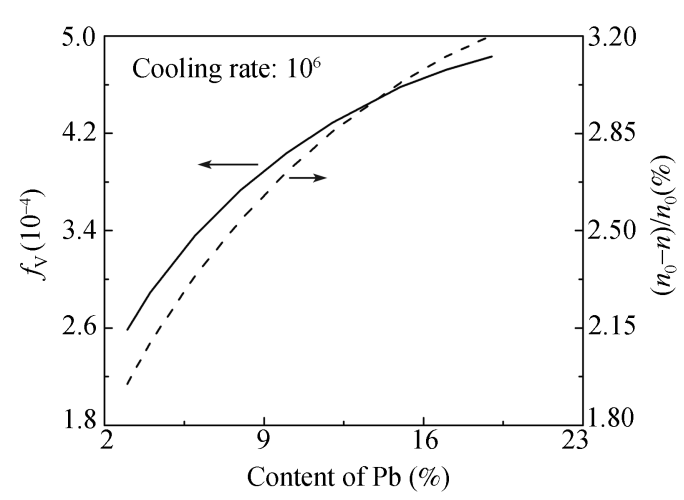

Figure 4 The volume fraction of the minority phase droplets as a function of the $\mathrm{Pb}$ content of the alloy when $n / n_{0}$ arrives at $0.9 . n_{0}$ is the number density calculated by neglecting the interaction between droplets.

Figure 4 shows the volume fraction and the number density of the minority phase droplets as a function of the $\mathrm{Pb}$ content of the alloy. For a given cooling rate, the volume fraction of the droplets increases and the interaction between the droplets becomes stronger with the $\mathrm{Pb}$ content.

\section{Conclusions}

The microstructure evolution during a liquid-liquid phase transformation in a rapidly cooled $\mathrm{Al}-\mathrm{Pb}$ alloy was calculated. The results show that the interaction between the minority phase droplets has a visible effect on the nucleation of the minority phase droplets. For a given alloy composition, its effect increases with the cooling rate. For a given cooling rate, its effect increases with the content of $\mathrm{Pb}$.

1 An J, Dong C, Zhang Q Y. Improvement of the wear behavior of stircast Al-Si-Pb alloys by hot extrusion. Tribol Int, 2003, 36: $25-34$

2 Liu Y, Guo J J, Su Y Q, et al. Numerical simulation of coarsening of second phase during rapid cooling an immiscible alloys through the miscibility gap. Trans Nonferrous Met Soc China, 2001, 11: 84-89

$3 \mathrm{Lu} \mathrm{X} \mathrm{Y,} \mathrm{Cao} \mathrm{C} \mathrm{D,} \mathrm{Kolbe} \mathrm{M,} \mathrm{et} \mathrm{al.} \mathrm{Microstructure} \mathrm{analysis} \mathrm{of} \mathrm{Co-Cu} \mathrm{alloys} \mathrm{undercooled} \mathrm{prior} \mathrm{to} \mathrm{solidification.} \mathrm{Mat} \mathrm{Sci} \mathrm{Eng} \mathrm{A,}$ 
2004, 375-377: 1101-1104

4 Chen G Y, Tian C, Yang L, et al. The study of the manufacturing of steel/Al-Pb composite bearing material by spray forming. Chin J Mech, 2002, 38(8): 139-142

5 Zhu M, Gao Y, Chung C Y, et al. Improvement of the wear behaviour of Al-Pb alloys by mechanical alloying. Wear, 2000, 242: $47-53$

6 Carberg T, Fredriksson H. The influence of microgravity on the structure of Zn-Bi immiscible alloys. Metal Trans, 1982, 11: $1665-1676$

7 Granasy L, Ratake L. Homogeneous nucleation within the liquid miscibility cap of Zn-Pb alloys. Scripta Metallurgica, 1993, 28: $1329-1334$

8 Zhao J Z, Ratke L, Feuerbacher B. Microstructure evolution of immiscible alloys during cooling through the miscibility gap. Modelling Simul Mater Sci Eng, 1998, 6: 123-139

9 Zhao J Z, Ratke L. Repeated nucleation of minority phase droplets during cooling through miscibility gap. Scripta Materialia, 1998, 39: 181-188

10 Zhao J Z. Modeling and simulation of the microstructure evolution during a cooling of immiscible of immiscible alloys in the miscibility gap. J Mater Sci Tech, 2002, 3(18): 197-205

11 Zhao J Z, Gao L L. Effect of Brownian coagulation on the liquid-liquid decomposition in gas-atomized alloy drops. J Mater Sci Tech, 2006, 22(3): 321-323

12 Zhao J Z. Formation of the minor phase shell on the surface of hypermonotectic alloy powders. Scripta Materialia, 2006, 54: $247-250$

13 Zener C. Theory of growth of spherical precipitates from solid solutions. J Appl Phys, 1949, 20: 950-953

14 Lifshttz I M, Slyozov V V. The kinetics of precipitation from supersaturated solid sloutions. J Phys Chem Solids, 1961, 19(1): $35-50$

15 Marqusee J A, Ross J. Theory of Ostwald ripening: Competitive growth and its dependence on volume fraction. J Chem Phys, 1984, 80: 536-543 